Calculating expectations with time-dependent perturbations in Quantum Monte Carlo

\author{
M. H. Kalos \\ Lawrence Livermore National Laboratory \\ Livermore, CA 94550 \\ F. A. de Saavedra \\ Departamento de Física Moderna, \\ Facultad de Ciencias \\ Granada, Spain, E-18071
}

May 2004

Journal of Chemical Physics 
This document was prepared as an account of work sponsored by an agency of the United States Government. Neither the United States Government nor the University of California nor any of their employees, makes any warranty, express or implied, or assumes any legal liability or responsibility for the accuracy, completeness, or usefulness of any information, apparatus, product, or process disclosed, or represents that its use would not infringe privately owned rights. Reference herein to any specific commercial product, process, or service by trade name, trademark, manufacturer, or otherwise, does not necessarily constitute or imply its endorsement, recommendation, or favoring by the United States Government or the University of California. The views and opinions of authors expressed herein do not necessarily state or reflect those of the United States Government or the University of California, and shall not be used for advertising or product endorsement purposes. 


\title{
Calculating expectations with time-dependent perturbations in Quantum Monte Carlo
}

\author{
M. H. Kalos \\ Lawrence Livermore National Laboratory \\ Livermore, California 94550 \\ F. Arias de Saavedra \\ Departamento de Física Moderna, \\ Facultad de Ciencias \\ Granada, Spain, E-18071
}

(Dated: May 27th, 2004)

\begin{abstract}
We show that a small perturbation periodic in imaginary time can be used to compute expectation values of non-differential operators that do not commute with the Hamiltonian within the framework of Quantum Diffusion Monte Carlo. Some results for the harmonic oscillator and the helium atom are presented showing the validity of the proposed method.
\end{abstract}




\section{INTRODUCTION}

Quantum Monte Carlo (QMC) comprises different approaches for solving the Schrödinger equation of a system using stochastic techniques. QMC usually represents the state under study by means a set of positions in a $3 N$-dimensional space with a probability distribution proportional to the wave function. This representation combined with a trial wave function $\psi_{T}$ for a bosonic systems enables the calculation of the energy and expectations of operators that commute with the Hamiltonian.

QMC techniques have been successfully applied to a great variety of system as nuclei [1], molecules [2], atoms [3] and solids [4]. Nevertheless there are still open problems as the sign problem for fermionic systems, and the unbiased calculation of expectations for operators that do not commute with the Hamiltonian.

Since the introduction of the extrapolation method [5], much effort has been invested in finding unbiased estimators of the expectation values of operators. They can be basically classified in two different strategies. A general approach involves estimating the ratio of the exact wave function $\psi$ to $\psi_{T}$. This ratio combined with a "mixed estimator" gives an approximation to $\psi^{2}$. A second approach samples $\psi^{2}$ by correlating the configurations for pairs of walkers.

The first strategy has been more extensively used and is based on the fact that $\psi / \psi_{T}$ is proportional to the asymptotic value of the total offspring of a walker after branching over many time steps. [6]. One version is the "future walking method," another the time correlation method. The future walking method [7]-[9] can use tagging algorithms to calculate the asymptotic number of descendants. The tagging process can be eliminated by keeping a weight proportional to the progeny of every walker $[10,11]$ making the method simpler to implement. This method has been has been successfully applied to some problems to provide very accurate results [7] -[13]. The reptation Quantum Monte Carlo method [14] is another method that is equivalent to the future walking method carrying weights in the part concerning to the calculation of expectations. The time correlation method has been applied with good results in the context of path integral Monte Carlo [15].

The second strategy is called Bilinear Quantum Monte Carlo [16, 17]. In this method, the use of correlated pairs of walkers instead of walkers samples $\psi^{2}$ by using the marginal distribution of walkers. This method has been used within the context of Green's function 
Monte Carlo [16] and of the Diffusion Monte Carlo [17]. The method is also capable of calculating differences of energy between very similar systems with small variances. Although the results provided by this method are valuable, it remains more complicated than future walking or time correlation methods.

We propose here a very simple alternative to the methods mentioned above to calculate expectations of non-differential operators. We will see that adding an appropriate perturbation periodic in imaginary time to the Hamiltonian of our system, makes it possible to calculate the required results.

The structure of the paper is the following. In Section II, we present the theoretical basis of our idea for calculating expectations in some detail showing how to implement it in a diffusion Monte Carlo code. In Section III, we present the results for a harmonic oscillator and for the Helium atom. Finally we offer our conclusions in Section IV.

\section{DESCRIPTION OF THE METHOD}

Let us recall how Diffusion Monte Carlo (DMC) works. For a system with Hamiltonian, $H$, the imaginary time Schrödinger equation will be

$$
\frac{\partial \psi(\vec{R}, \tau)}{\partial \tau}+H \psi(\vec{R}, \tau)=0
$$

We expand $\psi(\vec{R}, \tau)$ in terms of the eigenfunctions of $H, \phi_{k}(\vec{R})$, with eigenvalues $E_{k}, k=$ $0,1, \ldots$ labeled with $E_{k}<E_{l}$ if $k<l$

$$
\psi(\vec{R}, \tau)=\sum_{k=0}^{\infty} c_{k}(\tau) \phi_{k}(\vec{R}) .
$$

We substitute into the first equation to obtain:

$$
\sum_{k=0}^{\infty}\left(c_{k}^{\prime}(\tau)+E_{k} c_{k}(\tau)\right) \phi_{k}(\vec{R})=0
$$

The solutions of the previous equations are

$$
c_{l}(\tau)=c_{l} e^{-E_{l} \tau}
$$

with $c_{l}$ specifying the initial conditions. DMC is able to describe the ground state since for imaginary time values much bigger that the relaxation time, $\tau_{0}=1 /\left(E_{1}-E_{0}\right)$

$$
e^{E_{0} \tau} \psi(\vec{R}, \tau) \quad \underset{\tau \gg \tau_{0}}{\longrightarrow} c_{0} \phi_{0}(\vec{R})
$$


This means that asymptotically only the projection on the ground state survives.

Our goal is the calculation of the expectation value of a non-differential operator, $A(\vec{R})$, in the ground state of the system under study. To get this, we analyze the imaginary time Schrödinger equation of a hypothetical system with Hamiltonian:

$$
H_{\lambda}=H+\lambda(\tau) A(\vec{R})
$$

with $\lambda(\tau)$ function of the imaginary time, $\tau$. Since we have in mind performing a perturbative analysis in terms of $H$, we assume that this function is very small. Later we demonstrate that this is not a limit to the accuracy of our method. The quantum Monte Carlo treatment of perturbations independent of imaginary time has been studied in the paper by Caffarel and Hess [18] where they obtain perturbative estimations of the interaction energy of molecules.

The imaginary time Schrödinger equation for this system is now

$$
\frac{\partial \psi_{\lambda}(\vec{R}, \tau)}{\partial \tau}+H \psi_{\lambda}(\vec{R}, \tau)+\lambda(\tau) A(\vec{R}) \psi_{\lambda}(\vec{R}, \tau)=0
$$

Using Eq. (2) for $\Psi_{\lambda}$, renaming the coefficients $c_{\lambda k}(\tau)$ and substituting into Eq. (7), we obtain:

$$
\sum_{k=0}^{\infty}\left(c_{\lambda k}^{\prime}(\tau) \phi_{k}(\vec{R})+E_{k} c_{\lambda k}(\tau) \phi_{k}(\vec{R})+c_{\lambda k}(\tau) \lambda(\tau) A(\vec{R}) \phi_{k}(\vec{R})\right)=0
$$

If we multiply the last equation by $\phi_{l}^{*}(\vec{R})$ and integrate over $\vec{R}$ we finally find:

$$
c_{\lambda l}^{\prime}(\tau)+E_{l} c_{\lambda l}(\tau)+\lambda(\tau) \sum_{k=0}^{\infty} c_{\lambda k}(\tau) A_{l k}=0 \quad l=0,1, \ldots
$$

where

$$
A_{l k}=\int d \vec{R} \phi_{l}^{*}(\vec{R}) A(\vec{R}) \phi_{k}(\vec{R})
$$

As we have already mentioned, we assume that $\lambda(\tau)$ is very small, so we need solve Eq.(9) considering only the leading order in the magnitude of $\lambda(\tau)$. This can be accomplished by taking $c_{\lambda k}(\tau)=c_{k}(\tau)$ in all the terms multiplied by $\lambda(\tau)$. Under these assumptions, the solution of the equations are

$$
c_{\lambda l}(\tau)=c_{l} e^{-E_{l} \tau}-e^{-E_{l} \tau} \sum_{k=0}^{\infty} c_{k} A_{l k} \int_{0}^{\tau} d \tau^{\prime} e^{\left(E_{l}-E_{k}\right) \tau^{\prime}} \lambda\left(\tau^{\prime}\right)
$$

where, in order to keep the same initial conditions as when the perturbation is not present, we have put $\lambda(0)=0$. The terms not considered in the previous solutions involve at least $\lambda^{2}(\tau)$ 
If at very large imaginary time we subtract it from the unperturbed result, we obtain

$$
e^{E_{0} \tau}\left(\psi(\vec{R}, \tau)-\psi_{\lambda}(\vec{R}, \tau)\right) \quad \underset{\tau \gg \tau_{0}}{\longrightarrow} \phi_{0}(\vec{R}) \sum_{k=0}^{\infty} c_{k} A_{0 k} \int_{0}^{\tau} d \tau^{\prime} e^{\left(E_{0}-E_{k}\right) \tau^{\prime}} \lambda\left(\tau^{\prime}\right)
$$

This difference has a somewhat complicated structure. In order to simplify it, we propose a particular choice of $\lambda(\tau)$, namely

$$
\lambda(\tau)=\lambda_{0} \sin (\omega \tau)
$$

with $\lambda_{0}$ a constant that controls the size of the perturbation. So for this choice of the perturbation and in the long time regime, we can write:

$$
e^{E_{0} \tau}\left(\psi(\vec{R}, \tau)-\psi_{\lambda}(\vec{R}, \tau)\right) \underset{\tau \gg \tau_{0}}{\longrightarrow} \phi_{0}(\vec{R})\left(\sum_{k=0}^{\infty} \frac{c_{k} A_{0 k} \lambda_{0} \omega}{\left(E_{k}-E_{0}\right)^{2}+\omega^{2}}-\frac{c_{0} A_{00} \lambda_{0}}{\omega} \cos (\omega \tau)\right)
$$

We can see that the infinite sum has no dependence on imaginary time so we can eliminate its contribution by subtracting the mean over some number of periods of the perturbation. After that there is still a periodic component in imaginary time which contains the expectation value of the operator we are interested in for the ground state of the unperturbed Hamiltonian. Since we have subtracted the average, this periodic component has zero mean but we can extract $A_{00}$ using the root-mean-square deviation as we will discuss later.

The motivation for using a sinusoidal perturbation is simple: we want the average perturbation, reflected in an average weight to be zero, so that the perturbed and unperturbed system do not diverge, with the perturbation always remaining small. Thus the Monte Carlo statistics of the expectation or perturbation improve homogeneously as the calculation proceeds.

Both the future walking method carrying weights $[10,11]$ and the reptation quantum Monte Carlo method [14] may be interpreted within this context as taking $\lambda(\tau)=\lambda_{0}$. It can be argued that this choice of perturbation does not satisfy $\lambda(0)=0$ but this is not a problem since we are interested in the limit of vanishing perturbation. For this choice of perturbation, Eq. (12) will be:

$$
e^{E_{0} \tau}\left(\psi(\vec{R}, \tau)-\psi_{\lambda}(\vec{R}, \tau)\right) \underset{\tau \gg \tau_{0}}{\longrightarrow} \phi_{0}(\vec{R})\left(c_{0} A_{00} \lambda_{0} \tau+\sum_{k=1}^{\infty} \frac{c_{k} A_{0 k} \lambda_{0}}{E_{k}-E_{0}}\right)
$$

where we can see that the difference of the wave functions diverges at long time. This divergence can be eliminated by dividing by $\lambda_{0} \tau$ so that the average is $A_{00}$ in the long time regime. 
For practical applications using DMC, it is crucial to use importance sampling in order to reduce the statistical fluctuations to a reliable level. That is, we solve the imaginary time Schrödinger equation for

$$
f(\vec{R}, \tau)=e^{E_{T} \tau} \psi_{T}(\vec{R}) \psi(\vec{R}, \tau)
$$

with $\psi_{T}(\vec{R})$ a trial wave function for our system. The last equation can be solved in the form:

$$
f\left(\vec{R}^{\prime}, \tau+d \tau\right)=\int d \vec{R} G\left(\vec{R}^{\prime}, \vec{R}, d \tau\right) f(\vec{R}, \tau)
$$

with $G\left(\vec{R}^{\prime}, \vec{R}, d \tau\right)$ Green's function $\exp (-H \tau)$, which for very small $d \tau$ and for Hamiltonian in the form

$$
H=-\frac{1}{2} \nabla_{\vec{R}}^{2}+V(\vec{R})
$$

can be approximated neglecting $(d \tau)^{3}[19]$ as

$$
G\left(\vec{R}^{\prime}, \vec{R}, d \tau\right)=\frac{\exp \left\{-\frac{\left(\vec{R}^{\prime}-\vec{R}-d \tau\left(\vec{F}(\vec{R})+\vec{F}\left(\vec{R}^{\prime}\right)\right) / 2\right)^{2}}{2 d \tau}-\left[\frac{E_{L}(\vec{R})+E_{L}\left(\vec{R}^{\prime}\right)}{2}-E_{T}\right] d \tau\right\}}{(2 \pi d \tau)^{3 N / 2}}
$$

Here $N$ is the number of particles in the system, $E_{L}(\vec{R})$ is the local energy

$$
E_{L}(\vec{R})=\frac{H \psi_{T}(\vec{R})}{\psi_{T}(\vec{R})}
$$

and $\vec{F}(\vec{R})$ is the drift force

$$
\vec{F}(\vec{R})=\frac{\nabla_{\vec{R}} \psi_{T}(\vec{R})}{\psi_{T}(\vec{R})}
$$

To use Eq. (19), one begins with a walker at $\vec{R}$ and calculates its local energy. The corresponding drift is calculated, generating

$$
\vec{R}_{1}=\vec{R}+\frac{d \tau}{2} \vec{F}(\vec{R})
$$

One samples a displacement $\vec{T}$ randomly from the $3 N$ Gaussian distribution with zero mean and variance $d \tau$ and puts

$$
\vec{R}_{2}=\vec{R}_{1}+\vec{T}
$$

According to Eq. (19)

$$
\vec{R}_{2}=\vec{R}^{\prime}-\frac{d \tau}{2} \vec{F}\left(\vec{R}^{\prime}\right)
$$

so since the displacement is small one can approximate the new position as

$$
\vec{R}^{\prime}=\vec{R}_{2}+\frac{d \tau}{2} \vec{F}\left(\vec{R}_{2}\right)
$$


The local energy is calculated at the new position to give the branching of the walker as

$$
e^{-\left(\left(E_{L}(\vec{R})+E_{L}\left(\vec{R}^{\prime}\right)\right) / 2-E_{T}\right) d \tau}
$$

When the same analysis is performed for the perturbed Hamiltonian, we observe that, since $A(\vec{R})$ is not a differential operator, the diffusion part (sampling and drifting) of the walk is not modified as compared with the unperturbed system. The only modifications caused by the perturbation are on the branching factor. This now becomes

$$
e^{-\left(\left(E_{L}(\vec{R})+E_{L}\left(\vec{R}^{\prime}\right)\right) / 2-E_{T}\right) d \tau} e^{-\left(A(\vec{R}) \int_{\tau}^{\tau+d \tau / 2} \lambda\left(\tau^{\prime}\right) d \tau^{\prime}+A\left(\vec{R}^{\prime}\right) \int_{\tau+d \tau / 2}^{\tau+d \tau} \lambda\left(\tau^{\prime}\right) d \tau^{\prime}\right)}
$$

where we can see that in addition to the branching factor of the unperturbed system, there is an extra branching that derives from the perturbation. The idea is to perform a walk for the unperturbed system and to store separately an accumulated weight that reflects one minus the branching caused by the perturbation for every walker. We can accumulate this quantity and then for our choice of perturbation take the limit of vanishing perturbation. The branching caused in one step for a walker is:

$$
B_{\lambda_{0}}\left(\vec{R}, \vec{R}^{\prime} ; \tau, d \tau\right)=e^{-\lambda_{0} w\left(\vec{R}, \vec{R}^{\prime}\right)}
$$

where we have separated $\lambda_{0}$ and we have defined

$$
w\left(\vec{R}, \vec{R}^{\prime}\right)=A(\vec{R}) \int_{\tau}^{\tau+d \tau / 2} \sin \left(\omega \tau^{\prime}\right) d \tau^{\prime}+A\left(\vec{R}^{\prime}\right) \int_{\tau+d \tau / 2}^{\tau+d \tau} \sin \left(\omega \tau^{\prime}\right) d \tau^{\prime}
$$

We examine the information carried by the walker $\vec{R}^{\prime}$ after the first step. This is $1-$ $B_{\lambda_{0}}\left(\vec{R}, \vec{R}^{\prime} ; 0, d \tau\right)$. We can divide this by $\lambda_{0}$ and then take the limit $\lambda_{0} \rightarrow 0$, yielding

$$
\lim _{\lambda_{0} \rightarrow 0} \frac{1-B_{\lambda_{0}}\left(\vec{R}, \vec{R}^{\prime} ; 0, d \tau\right)}{\lambda_{0}}=w\left(\vec{R}, \vec{R}^{\prime}\right)
$$

If in the following step, the walker becomes $\vec{R}^{\prime \prime}$, the quantity to be carried is now $1-$ $B_{\lambda_{0}}\left(\vec{R}, \vec{R}^{\prime} ; 0, d \tau\right) B_{\lambda_{0}}\left(\vec{R}^{\prime}, \vec{R}^{\prime \prime} ; d \tau, d \tau\right)$. Again dividing by $\lambda_{0}$, we can take $\lambda_{0}$ to zero obtaining $w\left(\vec{R}, \vec{R}^{\prime}\right)+w\left(\vec{R}^{\prime}, \vec{R}^{\prime \prime}\right)$. From this, we see that we can calculate exactly the limit of small perturbations if, instead of multiplying the $B_{\lambda_{0}}$ and subtracting it from one, we simply sum the corresponding $w$. So if walker $\vec{R}_{0}$ becomes $\vec{R}_{k}$ after $k=1, \ldots, n$ steps the accumulated quantity to be carried by $\vec{R}_{n}$ is:

$$
W_{n}\left(\vec{R}_{n} ; n d \tau\right)=\sum_{k=1}^{n} w\left(\vec{R}_{k-1}, \vec{R}_{k}\right)
$$


If we take the mean of this quantity over the entire random walk for all the walkers in the population, we obtain a periodic function of imaginary time. This function is the DMC counterpart of Eq. (14) divided by $c_{0} \phi_{0}(\vec{R}) \lambda_{0}$. In order to extract $A_{00}$ from this, we must first subtract the mean with respect to imaginary time for a certain number of periods and calculate their standard deviation. This will be $A_{00} / \sqrt{2} \omega$.

The possibility of carrying out the exact limit of small perturbations is an important simplification for our method since there is now no question whether $\lambda_{0}$ is small enough to satisfy our analysis. Our perturbation has therefore only one free parameter, $\omega$. Another fact that reduces the time of computation of our method is that we can use the same walk to calculate different expectations by simply storing separately the corresponding weights of the operators under study. That is, we do not have to make a separate walk for every expectation to be calculated for the system.

\section{RESULTS}

We begin this section discussing some results for the harmonic oscillator. This system has a Hamiltonian that, using reduced units [17], can be written as:

$$
H=-\frac{1}{2} \nabla_{\vec{R}}^{2}+\frac{1}{2} \vec{R}^{2}
$$

We have first applied our method to this system for two reasons: its simplicity and the fact that its time--dependent Green function is analytically known [20]. This has the form:

$$
G\left(\vec{R}^{\prime}, \vec{R}, d \tau\right)=\left(\frac{1}{2 \pi \sinh d \tau}\right)^{N / 2} \exp \left(-\frac{\vec{R}^{2}+\vec{R}^{\prime 2}}{2 \tanh d \tau}+\frac{\vec{R} \cdot \vec{R}^{\prime}}{\sinh d \tau}\right)
$$

with $N$ the number of dimensions of the system which can have any value. If we use as importance function

$$
\psi_{T}(\vec{R})=e^{-\frac{1}{2} \alpha \vec{R}^{2}}
$$

the time-dependent Green function becomes:

$$
G\left(\vec{R}^{\prime}, \vec{R}, d \tau\right)=\left(\frac{1}{2 \pi \beta}\right)^{N / 2} \exp \left(-\frac{\left(\vec{R}^{\prime}-\gamma^{-1} \vec{R}\right)^{2}}{2 \beta}\right)\left(\frac{e^{d \tau}}{\gamma}\right)^{N / 2} \exp \left(\frac{\beta\left(\alpha^{2}-1\right) \vec{R}^{2}}{2}\right)
$$

where we have used as $E_{T}=N / 2$ the exact energy of the ground state and have defined $\beta \gamma=\sinh d \tau$ with

$$
\gamma=\cosh d \tau+\alpha \sinh d \tau
$$


In the Green function formula, we can clearly see the sampling and the branching parts. The sampling part is a Gaussian with mean $\gamma^{-1} \vec{R}$ and variance $\beta$. Since $\alpha=1$ is the exact solution, we can also see that in this case the branching is unity, independent of the position of the walker.

In our first test for our method, we have used as operator $A(\vec{R})=\vec{R}^{2}$. The virial theorem tells us that $<\vec{R}^{2}>=N / 2$. Using the exact Green's function for the harmonic oscillator allows us to go beyond the short-time limit. We have set $N=1$ and have used $d \tau=0.1$, $\omega=0.01$ and populations of 8000 walkers and generating 10 blocks of 100000 steps. First we have calculated with $\alpha=1$. If we calculate, the mean of the accumulated weights caused by the perturbation, we get $0.5111(4)$. We can see that there is an important bias in this result. This bias can not be due to the time step since we are using the exact Green function nor to the fluctuations in the population size since this is constant. This bias is caused by the influence of the other states in the perturbed ground state as was discussed following Eq. (14). Accordingly, we subtracted the mean over twenty periods around every step; the standard deviation of the weights now gives $0.49986(32)$. This result is consistent with the exact value of 0.5 .

It is known that if the trial function is the exact ground state wavefunction, the mixed estimator would provide the exact expectation value even for operators that do not commute with the Hamiltonian. Since this is just the case of the previous result, a possible criticism to our example is that we are simply getting the result provided by the mixed estimator. We can demonstrate that this is not the case by simply using a different value of the parameter $\alpha$. Here, it is possible to calculate exactly the value of the mixed estimator of the operator, $1 /(1+\alpha)$. For $\alpha=0.95$ the mixed estimator is 0.513 . In the same conditions of the first calculation and after subtracting the mean, we present in Table 1 the results for different populations. They show that we find the exact expectation value instead of the mixed estimator value. Since we have carried out different calculations and we have always obtained expectations bigger than 0.5 , we can conclude that there is a small finite population bias. The result with $\infty$ is an extrapolation assuming that the finite population bias is proportional to $N_{w}^{-1}$ and is consistent with the exact value.

A second test of the validity of our method involves the helium atom. In the infinite 
nuclear mass approximation, this has a Hamiltonian

$$
H=-\frac{1}{2} \nabla_{\vec{R}}^{2}-\frac{2}{r_{1}}-\frac{2}{r_{2}}+\frac{1}{r_{12}}
$$

with $\vec{R}=\left(\vec{r}_{1}, \vec{r}_{2}\right)$. This Hamiltonian has singularities in the potential at the nucleus and when the two electrons coincide. The use of importance sampling is crucial. We have used a very simple trial function for this case that incorporates Kato's cusp conditions [21]. This is:

$$
\psi_{T}(\vec{R})=\exp \left(-2\left(r_{1}+r_{2}\right)+\frac{1}{2} r_{12}\right)
$$

which provides a drift force $\vec{F}(\vec{R})=\left(-2 \hat{r}_{1}+1 / 2 \hat{r}_{12},-2 \hat{r}_{2}-1 / 2 \hat{r}_{12}\right)$ and a local energy

$$
E(\vec{R})=-\frac{5}{4}+\left(\hat{r}_{1}-\hat{r}_{2}\right) \cdot \hat{r}_{12}
$$

We have performed some calculations using this trial function. In all of them, we have taken $d \tau=0.001$ since, as in our propagator we are neglecting terms in $(d \tau)^{3}$, we can consider that we are very close to the zero time step limit. In order not to have a very long block, we have calculated using $\omega=1$. Taking into account that the size of the blocks depends on $\omega d \tau$ and this is 0.001 as it was in the case of the harmonic oscillator, we have used blocks of 100000 steps. We show in Table 2 results for two calculations with population size of 8000 and 64000 walkers for different non-differential operators. For completeness, we also show the mean of the local energy using the classic mixed estimator.

If we compare the results provided by our method to very precise variational calculations for the helium atom [22] that can be considered exact to the digits given, we see that there are significant biases in all of them. We note that the bias becomes smaller as the population becomes bigger which indicates that it is a finite population effect. So, using the values shown in Table 2, and assuming that the bias is proportional to $N_{w}^{-1}$, we have extrapolated to an infinite population. The results from the extrapolation are also shown in the Table under the $\infty$ symbol. These results are consistent with the accurate variational results showing again the validity of our method.

\section{CONCLUSIONS}

We have shown that the use of a periodic perturbation on imaginary time multiplied by a non-differential operator permits the straightforward calculation of the expectation of that 
operator in the ground state of the system under study within the framework of DMC. We have applied this method to the harmonic oscillator and to the Helium atom verifying its validity.

\section{ACKNOWLEDGMENTS}

This work was performed under the auspices of the U.S. Department of Energy by the University of California, Lawrence Livermore National Laboratory under contract No. W7405-Eng.-48. F.A. is partly supported by the Spanish Ministerio de Ciencia y Tecnología in the project BFM2002-00200. We thank Kevin Schmidt for helpful comments and David Hardin for a critical reading of the text.

[1] S.C. Pieper, K. Varga and R.B. Wiringa, Phys. Rev. C 66, 044310 (2002); S.C. Pieper and R.B. Wiringa, Ann. Rev. Nucl. Part. Sci. 51, 53 (2001).

[2] M. Mella and J.B. Anderson, J. Chem. Phys. 118, 3102 (2003); J.B. Anderson, ibid. 112, $9699(2000)$.

[3] A. Aspuru-Guzik and W.A. Lester, Jr., in Handbook of Numerical Analysis, Vol. X, Special Volume: Computational Chemistry C. Le Bris, editor, Elsevier (2003).

[4] M. Foulkes, L. Mitas, R. Needs and G. Rajagopal, Rev. Mod. Phys. 73, 33 (2001).

[5] P.A. Whitlock, D.M. Ceperley, G.V. Chester, and M.H. Kalos, Phys. Rev. B 19, 5598 (1979).

[6] K.S. Liu, M.H. Kalos, and G.V. Chester, Phys. Rev. A 10, 303 (1974).

[7] P.J. Reynolds, R.N. Barnett, B.L. Hammond, and W.A. Lester, Jr., J. Stat. Phys. 43, 1017 (1986).

[8] R.N. Barnett, P.J. Reynolds, and W.A. Lester, Jr., J. Comput. Phys. 96, 258 (1991).

[9] K.J. Runge, Phys. Rev. B 45, 7229 (1992).

[10] J. Casulleras and J. Boronat, Phys. Rev. B 52, 3654 (1995).

[11] P. Langfelder, S.M. Rothstein, and J. Vrbik, J. Chem. Phys. 107, 8525 (1997)

[12] A. Sarsa, J. Boronat and J. Casulleras, J. Chem. Phys. 116, 5956 (2002)

[13] M. Hornik and S.M. Rothstein, in Recent Advances in Quantum Monte Carlo methods, Part II, W.A. Lester Jr, S.M. Rothstein and S. Tanaka editors, World Scientific (2002); M. Hornik, 
M. Snajdr, and S.M. Rothstein, J. Chem. Phys. 113, 3496 (2000).

[14] S. Baroni and S. Moroni, Phys. Rev. Lett. 82, 4745 (1999)

[15] A. Sarsa, K.E. Schmidt, and W.R. Magro, J. Chem. Phys. 113, 1366 (2000).

[16] S. Zhang and M.H. Kalos, J. of Stat. Phys. 70, 515 (1993).

[17] F. Arias de Saavedra and M.H. Kalos, Phys. Rev. E 67, 026708 (2003).

[18] M. Caffarel and O. Hess, Phys. Rev. A 43, 2138 (1991)

[19] S.A. Chin, Phys. Rev. A 42, 6991 (1990).

[20] R.P. Feynman, Statistical Mechanics: A Set of Lectures (Addison-Wesley, Reading, 1992).

[21] T. Kato. Commun. Pure and Appl. Math. 10,151 (1957).

[22] F. Arias de Saavedra, I. Porras, E. Buendía and F.J. Gálvez, J. Phys. B 28, 3123 (1995). 


\section{Tables}

\begin{tabular}{|c|}
\hline Population $<\vec{R}^{2}>$ \\
\hline $80000.5011(8)$ \\
\hline $320000.50003(30)$ \\
\hline$\infty 0.4997(7)$ \\
\hline
\end{tabular}

TABLE I: Twice the potential energy results for the monodimensional harmonic oscillator with different populations. The exact result is 0.5 .

\begin{tabular}{|c|c|c|c|c|}
\hline Population & 8000 & 64000 & $\infty$ & Exact \\
\hline$E$ & $2.90346(18)$ & $-2.90368(11)$ & $-2.9037(1)$ & -2.9037 \\
\hline$<V>$ & $-5.881(22)$ & $-5.8225(49)$ & $-5.814(9)$ & -5.8074 \\
\hline$<r_{1}^{-1}>$ & $1.737(15)$ & $1.6948(17)$ & $1.689(4)$ & 1.688 \\
\hline$<r_{1}>$ & $0.950(6)$ & $0.9305(9)$ & $0.928(2)$ & 0.929 \\
\hline$<r_{12}^{-1}>$ & $0.964(4)$ & $0.9459(9)$ & $0.943(2)$ & 0.946 \\
\hline$<r_{12}>$ & $1.451(7)$ & $1.4258(17)$ & $1.422(3)$ & 1.422 \\
\hline
\end{tabular}

TABLE II: Some results for the helium atom for different populations compared to the exact (in the digits shown) variational calculation from [22]. All the calculations, use $\omega=1$.

This work was performed under the auspices of the U.S. Department of Energy by University of California, Lawrence Livermore National Laboratory under Contract W-7405-Eng-48. 